manuscript No.

(will be inserted by the editor)

\title{
Energy Efficiency of Some Non-Cooperative, Cooperative and Hybrid Communication Schemes in Multi-Relay WSNs
}

\author{
Marcos Kakitani · Glauber Brante • \\ Richard Demo Souza • Anelise \\ Munaretto • Muhammad Ali Imran
}

Received: date / Accepted: date

\begin{abstract}
In this paper we analyze the energy efficiency of single-hop, multihop, cooperative selective decode-and-forward, cooperative incremental decodeand-forward, and even the combination of cooperative and non-cooperative schemes, in wireless sensor networks composed of several nodes. We assume that, as the sensor nodes can experience either non line-of-sight or some lineof-sight conditions, the Nakagami-m fading distribution is used to model the wireless environment. The energy efficiency analysis is constrained by a target outage probability and an end-to-end throughput. Our results show that in most scenarios cooperative incremental schemes are more energy efficient than the other methods.
\end{abstract}

\section{Introduction}

Wireless sensor networks (WSNs) are composed of a large number of sensor nodes that can have minimum dimensions and are placed inside or close to a phenomenon of interest. As it is not required from sensor nodes to be

This work was partially supported by CNPq and CAPES (Brazil).

M. Kakitani (corresponding author), G. Brante, R. D. Souza and A. Munaretto

CPGEI - Federal University of Technology - Paraná (UTFPR)

Av. Sete de Setembro, 3165

Curitiba, PR, Brazil

80230-901

Ph: +55 $4133104700 ;$ Fax: +55 4133104683

E-mail: mtkakitani@ieee.com, gbrante@ieee.org, richard@utfpr.edu.br, anelise@utfpr.edu.br

M. A. Imran

Centre for Communication Systems Research

University of Surrey

United Kingdom

GU2 7XH

E-mail: M.Imran@surrey.ac.uk 
placed in engineered or predetermined positions, they can be randomly deployed in inaccessible and irregular areas. This also demands sensor nodes to be supplied with self-organizing network protocols. These attributes provide WSNs a large range of applications, such as health, military and commercial. In health, sensor nodes can be applied to patients monitoring. In military, the rapid deployment and self-organizing characteristics make WSNs available for communications, surveillance and reconnaissance applications. In commercial applications, the use of WSNs can be related to inventory management, product quality control and disaster areas monitoring [1].

Sensor nodes are equipped with limited power sources for which recharging or replacement might not be possible. Thus, due to the battery lifetime limitations, the network lifetime is an important metric in WSNs. One of the frequently employed definitions is the time until the first node drains out its energy [32]. Thus, transmit power and overall energy consumption are major concerns in WSNs. For instance, the transmit power consumed by the nodes can be decreased if instead of performing long hops to a destination node, intermediate nodes are used to reach the destination by means of multiple shorter hops [16]. As transmit power is proportional to a power of the distance between two nodes [11], the multi-hop ( $\mathrm{MH})$ scheme requires each node to spend a smaller transmit power due to the decreased distance of each hop. Therefore, $\mathrm{MH}$ transmission can provide large transmit power savings if compared to the single-hop ( $\mathrm{SH}$ ) scheme in which the source communicates directly to the destination. Another possibility to reduce the required transmit power is the use of multiple transmit and/or receive antennas per node [3], also known as MIMO systems. If compared to single antenna communications, MIMO systems require less transmit power to achieve the same throughput, as shown in [21]. The use of multiple antennas provides spatial diversity, which reduces the harmful effects of fading by sending data through several independent paths [4]. Moreover, a new protocol is proposed in [27], which switches between the use of single or multiple antennas, depending on the channel condition, increases the energy efficiency of a wireless local area network.

However, WSNs can have size and cost restrictions which may preclude the use of multiple antennas at the sensor nodes. In addition, when practical energy consumption models are considered, as the number of antennas increases, no energy efficiency gains are obtained [12]. One alternative is the use of cooperative transmission (CT) schemes [26], [15], which can provide spatial diversity even with single antenna devices. Spatial diversity is achieved through the use of a partner relay node antenna. The partner node, due to the broadcast nature of the wireless medium, can overhear the source transmission and then forward the source frame to the destination node. The so called cooperative protocols define the specific behavior of the relay node. For instance, in amplify-andforward (AF) the relay node just amplifies the received signal and retransmits it to the destination node. In the compress-and-forward $(\mathrm{CF})$ strategy the relay node compress the received message, which is forwarded to the destination node [30]. In decode-and-forward (DF) the frame is decoded, re-encoded and then transmitted by the relay node. In the selective decode-and-forward (SDF) 
variant of the DF cooperative protocol the relay only forwards the frame to the destination if the decoded frame at the relay is error-free; otherwise the relay stays silent. The SDF protocol is shown to outperform both AF and DF in several scenarios [15], and requires less channel state information than CF. Moreover, the availability of a feedback channel is exploited by another variant of the DF protocol, the incremental decode-and-forward (IDF) cooperative protocol. The feedback channel provides the destination node a mechanism to indicate the relay node the failure or success of the source transmission. The relay only forwards the frame if it is successfully decoded at the relay, and if the destination requires so.

Although the MH transmission, multiple antennas and CT schemes can reduce the required transmit power of a sensor node, the power consumed by the transmitter and receiver circuitry of the sensor nodes is one aspect that should not be ignored in the energy efficiency analysis. For long distances the transmission energy represents the most relevant fraction of the total energy consumption. However, for short distance applications as WSNs the power consumed by the circuitry can be very relevant in the overall energy analysis [9]. In [10] a model comprising several building blocks of typical transmitter and receiver circuitry are modeled, including digital-to-analog and analog-to-digital converters, filters, frequency synthesizers and the power amplifier, among others. Following the definitions in [10] it is possible to adequately model the energy spent by both the transmitter and receiver circuitry. Moreover, note that when comparing schemes that employ different number of hops, the endto-end throughput should be considered, as the performance of a system could still be degraded even if a packet is received correctly, but with an excessive delay [17]. For instance, in a three-node scenario (source, relay, destination), $\mathrm{SH}$ requires only one time slot to transmit one frame while a half-duplex CT scheme requires two time slots. In order to achieve the same end-to-end throughput in both methods, the CT model should use twice the spectral efficiency of SH. For instance, in [7] we showed the importance of considering the circuitry consumption and the end-to-end throughput requirement in the energy efficiency analysis of a simple three-node network.

\subsection{Related Works}

Some related works, dealing with the comparison of different transmission schemes in wireless networks with multiple nodes can be found in the literature. For instance, the energy efficiency of two MH schemes in MIMO networks are compared in [18]. The two analyzed transmission schemes differ on the number of hops. The numerical results show that in many scenarios the transmission scheme that uses an increased number of hops requires less transmit power due to the shorter distance of each hop. However, the energy efficiency analysis does not take into account the total energy consumption, only the required transmit power is included. The end-to-end throughput is also not considered. Cooperative and non-cooperative schemes are studied in [20]. The SH, MH and 
SDF schemes have their bit error rate (BER) performance compared, with the SDF scheme achieving the best results. However, the analysis does not include the energy efficiency nor the end-to-end throughput restrictions.

In [13] CT and MH schemes are compared in a cellular network. The SDF cooperative scheme presents higher achievable rates than $\mathrm{MH}$ in scenarios composed of a small number of relays per cell. Nevertheless, neither the energy efficiency nor the end-to-end throughput are considered. The energy efficiency of IDF is studied in [24]. The analyzed scenarios are composed of one source node, one destination node and one relay node. Results show that for short distances between source and destination nodes $\mathrm{SH}$ is more energy efficient than IDF. However, spectral efficiency compensations in order to obtain the same end-to-end throughput are not imposed to the CT scheme. In [23] the energy efficiency of some hybrid relaying schemes, including AF/DF and CF/DF is analyzed under the constraint of a target outage probability. The proposed relaying strategy is able to obtain either transmit or receive diversity.

The system models of [18], [20] and [24] only consider non line-of-sight (NLOS) scenarios, while WSNs can often be under some line-of-sight (LOS) conditions [25]. Moreover, only incremental cooperation is considered in [24] and [23] and only SDF is studied in [20] and [13]. The comparison of IDF and SDF protocols could expose the effects of the availability of a feedback channel in the energy efficiency of cooperative communications. Another important issue is that the total number of nodes involved in the communication limits the number of possible transmission schemes between source and destination nodes. For instance, in a three-node scenario as in [24], only two non-cooperative (SH and $\mathrm{MH}$ with two hops) schemes are possible. However, if extended scenarios composed of several nodes are considered, a diverse number of transmission schemes can be constructed.

In this paper we analyze the energy efficiency of SH, MH, CT (SDF and IDF) and even hybrid models of non-cooperative and cooperative transmission schemes in WSNs with multiple nodes. The energy efficiency performance of the different schemes is analyzed under the constraint of a target outage probability and a target end-to-end throughput. The impact of a feedback channel and NLOS/LOS conditions are also considered. The objective is to determine the most appropriate transmission scheme in terms of energy efficiency under the above constraints.

\subsection{Main Contributions}

The energy efficiency analysis performed in this paper, under target outage and end-to-end throughput constraints, shows that the IDF cooperative and hybrid schemes have the best energy efficiency performances in most scenarios when compared with the other models. Considering the analyzed scenarios, only in case of some LOS and when the target end-to-end throughput is very high the cooperative and hybrid schemes may be outperformed by SH. Even though the increase in the number of relays in $\mathrm{MH}$ shortens the distance of each hop, the 
MH transmission presents the worst performance due to the required increased spectral efficiency at each hop and to the increased circuitry consumption. The $\mathrm{MH}$ scheme can outperform the other transmission methods when the endto-end throughput requirement is relaxed. However, in such cases either the maximum delay is considerably increased or the system complexity is much larger than that of SH or the CT schemes. To the best of our knowledge, there are no similar works that compare the energy efficiency performance of the cooperative and non-cooperative schemes considered in this paper, with an analysis of the impact of the end-to-end throughput requirements and also the impact of the number of available relays.

\subsection{Organization}

The rest of this paper is organized as follows. Section 2 presents the system model. Section 3 presents the outage probability and the energy consumption of the non-cooperative $\mathrm{SH}$ and $\mathrm{MH}$ schemes, the CT schemes and some hybrid transmission models. Section 4 presents numerical results, including the impact of the outage probability and end-to-end throughput requirements, as well as spatial reuse in $\mathrm{MH}$. Section 5 concludes the paper.

\section{System Model}

We consider a WSN where a source $(S)$ transmits a data frame to a destination $(D)$. We assume that there are $K$ intermediate nodes between $S$ and $D$, which can act as relays for $S$. The $k^{t h}$ relay is denoted as $R_{k}, k \in[1, K]$. For the sake of analytical tractability the $K+2$ nodes of interest are randomly disposed over a line, with $S$ at the origin, and $D$ at a distance $d$ from $S$. Such one-dimensional setup can be found, for instance, in production systems or highways. In the particular case of $K=0$ there are no relay nodes, and therefore only $\mathrm{SH}$ transmission is possible. For $K=1$ there is one relay between $S$ and $D$, and the number of possible transmission schemes is limited to three: $\mathrm{SH}, \mathrm{MH}$ and $\mathrm{CT}$. If the end-to-end throughput and target outage constraints are considered in this scenario, CT has better energy efficiency performance than $\mathrm{SH}$ and $\mathrm{MH}$, specially if a feedback channel is available [7]. For $K \geq 2$, we have a significant increase of possible transmission schemes. Besides the direct transmission from $S$ to $D$, we have the following options: i) MH transmission by means of shorter or longer hops with or without spatial reuse; ii) CT with or without the presence of a feedback channel (IDF or SDF, respectively); iii) hybrid schemes composed by a mix of cooperative and non-cooperative schemes. For all considered methods, the transmissions are orthogonal in time and the sensor nodes are half-duplex.

The Rayleigh distribution [11] is widely employed to model the multipath fading effects of the radio propagation environment, however it only models NLOS conditions. As the sensor nodes can often be under some LOS [25], the 
Rayleigh distribution is not necessarily the most suitable one. The Nakagami$m$ distribution [28] can be adapted to represent a wide variety of empirical measurements, while the severity of the fading can be adjusted by the parameter $m$. Based on the experimental results presented in [31], we consider $m=1$ for NLOS (equal to Rayleigh) and $m=2$ for the case of some LOS (less severe than Rayleigh). In addition, we consider that the channel is in long-term quasi-static fading. As the channel remains constant for a long period of time, simple retransmissions from $S$ are not very efficient because an entire set of retransmissions could suffer the same deep fading.

The energy efficiency is analyzed in terms of the total energy consumption per bit of each transmission scheme. Besides the required transmit power, that is distance dependent, we also consider the energy consumed by the transmit and receive circuitry. Following [10], the total consumed energy per bit in an $i$ to $j$ transmission $(i-j$ link) is:

$$
E_{b t, i j}=\frac{P_{P A, i j}+P_{T X}+P_{R X}}{R_{b}}
$$

where $P_{P A, i j}$ represents the power consumed by the power amplifier, $P_{T X}$ and $P_{R X}$ are respectively the transmitting and receiving power consumed by the internal RF circuitry, and $R_{b}$ corresponds to the bit rate in bits/s. Moreover, $R_{b}=\Delta \cdot B$, where $\Delta$ is the spectral efficiency and $B$ is the system bandwidth, in Hz. We consider the same block diagram for the RF circuitry introduced in [10], a model which is well accepted in the literature and is still up to date, as shown in [8]. For the transmitting circuitry, the following components are included: digital-to-analog converter, mixer, transmit filters and frequency synthesizer and the respective power consumptions are: $P_{D A C}, P_{m i x}, P_{f i l_{-} t x}$ and $P_{s y n}$, totalizing the consumed power for the transmitting circuitry as

$$
P_{T X}=P_{D A C}+P_{m i x}+P_{f i l \_t x}+P_{\text {syn }} .
$$

For the receiving circuitry, the following component blocks are considered: frequency synthesizer, low-noise amplifier, mixer, intermediate frequency amplifier, receive filter and analog-to-digital converter. The power consumptions are respectively: $P_{s y n}, P_{L N A}, P_{m i x}, P_{I F A}, P_{f i l_{-} t x}, P_{A D C}$, while the total power consumption for the receiving hardware is given by:

$$
P_{R X}=P_{s y n}+P_{L N A}+P_{m i x}+P_{I F A}+P_{f i l_{-} r x}+P_{A D C} .
$$

The power amplifier consumption depends on the amplifier efficiency [10], such that:

$$
P_{P A, i j}=\frac{\xi}{\eta} P_{i}
$$

where $\xi=3\left(\frac{\sqrt{M}-1}{\sqrt{M}+1}\right)$ is the peak-to-average ratio for an $M$-QAM modulation, $\eta$ is the amplifier drain efficiency and $P_{i}$ is the transmit power of node $i$. As we consider a narrowband single-carrier transceiver, typical of WSNs, the energy consumption of the baseband signal processing blocks is quite small when 
compared to the RF circuitry power consumption, and therefore is neglected in the model proposed in $[10]^{1}$.

The energy efficiency analysis is performed under the constraint of an outage probability. The outage probability is defined for an infinite block length code, however this assumption is not an invalidating issue, since there are several works in the literature showing that the outage probability can satisfactorily predict the frame error rate of good practical codes with relatively short block lengths $[2,14,19]$. An outage occurs in the transmission of a frame from nodes $i$ to $j$, when the SNR (Signal-to-Noise-Ratio) at node $j$ falls below a threshold value $\beta=2^{\Delta}-1$ [11]. Considering our specific system model, the frame received at node $j$ is given by:

$$
\mathbf{y}_{i j}=\sqrt{P_{i} \gamma_{i j}} h_{i j} \mathbf{x}+\mathbf{n}_{i j}
$$

where $\gamma_{i j}$ represents the path loss in the $i-j$ link, $h_{i j}$ is a scalar representing the Nakagami-m quasi-static fading, $\mathbf{x}$ corresponds to the transmitted frame and $\mathbf{n}_{i j}$ represents the AWGN vector, with variance $N_{0} / 2$ per dimension, where $N_{0}$ is the thermal noise power spectral density per Hz. The path loss between $i$ and $j$ is given by [11]:

$$
\gamma_{i j}=\left(\frac{G \lambda^{2}}{(4 \pi)^{2} d_{i j}^{\alpha} M_{l} N_{f}}\right),
$$

where $d_{i j}$ is the distance in meters between nodes $i$ and $j, \alpha$ represents the path loss exponent, $G$ is the total gain of the transmit and receive antennas, $\lambda$ is the wavelength, $M_{l}$ is the link margin and $N_{f}$ is the noise figure at the receiver. The instantaneous $\mathrm{SNR}$ in the $i-j$ link is:

$$
S N R_{i j}=\left|h_{i j}\right|^{2} \cdot \overline{S N R_{i j}}
$$

where $\overline{S N R_{i j}}=\frac{\gamma_{i j} P_{i}}{N}$ and $N=N_{0} \cdot B$ is the noise power spectral density. Finally, the outage probability of the $i-j$ link is given by [25]:

$$
\mathcal{O}_{i j}=\frac{\Psi\left(m, \frac{m N \beta}{\gamma_{i j} P_{i}}\right)}{\Gamma(m)},
$$

where $\Psi(a, b)=\int_{0}^{b} y^{a-1} \exp (-y) d y$ is the incomplete gamma function and $\Gamma(a)=\int_{0}^{\infty} y^{a-1} \exp (-y) d y$ is the complete gamma function. At high SNR, the incomplete gamma function can be approximated ${ }^{2}$ by $\Psi(a, b) \simeq(1 / a) \cdot b^{a}[25]$. Therefore:

$$
\mathcal{O}_{i j} \simeq \frac{1}{\Gamma(m+1)}\left(\frac{m N \beta}{\gamma_{i j} P_{i}}\right)^{m} .
$$

\footnotetext{
1 Note that the baseband processing can be neglected only because we assume a simple narrowband single-carrier transceiver. In the case of broadband multi-carrier transceivers, typical of local and wide area networks, then the baseband processing consumption has to be taken into account [6].

2 The high-SNR approximation is quite accurate for the outage values considered in this paper. For outage values lower than $10^{-2}$, the approximate and the exact curves match perfectly for $m=1$ and $m=2$, as shown in [29].
} 


\section{Transmission schemes}

In this section we present the outage probability, the optimal transmit power, and the total consumed energy per bit for $\mathrm{SH}, \mathrm{MH}, \mathrm{CT}$ and some hybrid transmission schemes.

\subsection{Single-hop Transmission (SH)}

In the SH scheme the communication involves only two nodes: $S$ and $D$, with node $S$ performing a direct transmission to node $D$. Note that in the SH transmission scheme the number of $R_{k}$ nodes is not relevant, since they are not used. Replacing $i$ and $j$ by $S$ and $D$ in (1), the total consumed energy per bit of the $\mathrm{SH}$ transmission is:

$$
E_{b t, S H}=E_{b t, S D}=\frac{P_{P A, S D}+P_{T X}+P_{R X}}{R_{b}} .
$$

In order to obtain the minimum consumed energy, $P_{P A, S D}$ must be minimized since $P_{T X}$ and $P_{R X}$ are not adjustable and depend on the specific technology. Considering $\mathcal{O}^{*}$ as the target end-to-end outage probability and substituting in (9), the $\mathrm{SH}$ optimal transmit power is:

$$
P_{S H}^{*}=\frac{m N \beta}{\gamma_{S D}\left[\Gamma(m+1) \mathcal{O}^{*}\right]^{1 / m}},
$$

such that $\mathcal{O}_{S H}=\mathcal{O}^{*}$.

\subsection{Multi-hop Transmission (MH)}

In the MH transmission scheme, the communication from $S$ to $D$ is accomplished through the use of relays positioned between them. Although the distance of each hop is shortened in the $\mathrm{MH}$ scheme with respect to the $\mathrm{SH}$ case, the number of hops in $\mathrm{MH}$ increases. In order to obtain the same end-to-end throughput of the $\mathrm{SH}$ model, in $\mathrm{MH}$ each node must operate at a spectral efficiency $L$ times that used in $\mathrm{SH}$, where $L$ is the number of hops between $S$ and $D$. Therefore, an outage event will occur when the received SNR falls below a threshold $\beta_{L}=2^{L \Delta}-1$. Thus, the outage probability for each link $i-j$ is:

$$
p_{i j} \simeq \frac{1}{\Gamma(m+1)}\left(\frac{m N \beta_{L}}{\gamma_{i j} P_{i}}\right)^{m} .
$$

The outage probability of the $\mathrm{MH}$ scheme is ${ }^{3}$ :

$$
\begin{aligned}
\mathcal{O}_{M H}= & p_{S R_{\mathcal{A}(1)}}+\left(1-p_{S R_{\mathcal{A}(1)}}\right) \cdot \sum_{w=1}^{W-1}\left(p_{R_{\mathcal{A}(w)} R_{\mathcal{A}(w+1)}} \cdot \prod_{z=1}^{w-1}\left(1-p_{\left.\left.R_{\mathcal{A}(z)} R_{\mathcal{A}(z+1)}\right)\right)}\right)\right. \\
& +\left(1-p_{S R_{\mathcal{A}(1)}}\right) \cdot p_{R_{\mathcal{A}(W)} D} \cdot \prod_{w=1}^{W-1}\left(1-p_{\left.R_{\mathcal{A}(w)} R_{\mathcal{A}(w+1)}\right)}\right.
\end{aligned}
$$

\footnotetext{
${ }^{3}$ Considering $\sum_{x=a}^{b}(\theta(x))=0$ if $a>b$ and $\prod_{x=a}^{b}(\theta(x))=1$ if $a>b$.
} 
where $\mathcal{A}$ is a vector that contains the relay nodes that are involved in the $\mathrm{MH}$ transmission, $\mathcal{A}(w)$ represents the $w^{\text {th }}$ element of $\mathcal{A}$, and $W$ is the cardinality of $\mathcal{A}$. For example, consider $K=5$ relays, and that only relays $R_{3}$ and $R_{4}$ are involved in the transmission. Then $\mathcal{A}(w)=[3,4]$, with $\mathcal{A}(1)=3, \mathcal{A}(2)=4$ and $W=2$.

The total consumed energy per bit is:

$$
\begin{aligned}
& E_{b t, M H}=p_{S R_{\mathcal{A}(1)}} \cdot \frac{P_{P A, M H}+P_{T X}+P_{R X}}{L R_{b}} \\
& +\left(1-p_{S R_{\mathcal{A}(1)}}\right) \cdot \sum_{w=1}^{W-1}\left(p_{R_{\mathcal{A}(w)} R_{\mathcal{A}(w+1)}} \cdot \frac{(w+1)\left(P_{P A, M H}+P_{T X}+P_{R X}\right)}{L R_{b}} .\right. \\
& \prod_{z=1}^{w-1}\left(1-p_{\left.\left.R_{\mathcal{A}(z)} R_{\mathcal{A}(z+1)}\right)\right)}\right. \\
& +\left(1-p_{S R_{\mathcal{A}(1)}}\right) \cdot \frac{(W+1)\left(P_{P A, M H}+P_{T X}+P_{R X}\right)}{L R_{b}} \text {. } \\
& \prod_{w=1}^{W-1}\left(1-p_{R_{\mathcal{A}(w)}} R_{\mathcal{A}(w+1)}\right) \text {. }
\end{aligned}
$$

The first term in (14) corresponds to the consumed energy if $R_{\mathcal{A}(1)}$ was not able to correctly decode the message from $\mathrm{S}$ and the frame is considered lost. The second term corresponds to successful decodings by the relay nodes until the transmission from $R_{\mathcal{A}(w)}$ to $R_{\mathcal{A}(w+1)}$, for $w<W$, which is not successfully decoded. Finally, the third term corresponds to the consumed energy if the message was correctly decoded by all relay nodes and then forwarded to $D$. All terms are divided by $L$ because, as spectral efficiency is multiplied by $L$, each single transmission is $L$ times faster than in $\mathrm{SH}$.

The outage probability in (13) can be rewritten as:

$$
\begin{aligned}
\mathcal{O}_{M H}= & \frac{f_{S R_{\mathcal{A}(1)}}}{\left(P_{M H}\right)^{m}}+\left(1-\frac{f_{S R_{\mathcal{A}(1)}}}{\left(P_{M H}\right)^{m}}\right) . \\
& \sum_{w=1}^{W-1}\left(\frac{f_{R_{\mathcal{A}(w)} R_{A(w+1)}}}{\left(P_{M H}\right)^{m}} \cdot \prod_{z=1}^{w-1}\left(1-\frac{\left.\left.f_{R_{\mathcal{A}(z)} R_{\mathcal{A}(z+1)}}\right)\right)}{\left(P_{M H}\right)^{m}}\right)\right. \\
& +\left(1-\frac{f_{S R_{\mathcal{A}(1)}}}{\left(P_{M H}\right)^{m}}\right) \cdot \frac{f_{R_{\mathcal{A}(W)} D}}{\left(P_{M H}\right)^{m}} \cdot \prod_{w=1}^{W-1}\left(1-\frac{f_{R_{\mathcal{A}(w)} R_{\mathcal{A}(w+1)}}}{\left(P_{M H}\right)^{m}}\right),
\end{aligned}
$$

where $f_{S R_{\mathcal{A}(1)}}=\frac{1}{\Gamma(m+1)}\left(\frac{m N \beta_{L}}{\gamma_{S R_{\mathcal{A}}(1)}}\right)^{m}, f_{R_{i} R_{j}}=\frac{1}{\Gamma(m+1)}\left(\frac{m N \beta_{L}}{\gamma_{R_{i} R_{j}}}\right)^{m}$ and $f_{R_{\mathcal{A}(W)} D}=$ $\frac{1}{\Gamma(m+1)}\left(\frac{m N \beta_{L}}{\gamma_{R_{\mathcal{A}}(W)^{D}}}\right)^{m}$. The optimal transmit power $P_{M H}^{*}$ can be obtained by replacing $\mathcal{O}_{M H}$ by a target outage probability $\mathcal{O}^{*}$ in (15) and calculating the smallest real and positive solution as a function of $P_{M H}$.

The performance of $\mathrm{MH}$ can be improved if spatial reuse is applied. For instance, consider the case of $K=2$. With spatial reuse, in a given time slot $S$ transmits to $R_{1}$ while $R_{2}$ transmits to $D$. In the next slot $R_{1}$ transmits to 
$R_{2}$. Note that in the first slot $S$ interferes in the transmission from $R_{2}$ to $D$ and $R_{2}$ interferes in the transmission from $S$ to $R_{1}$. Considering that all nodes transmit with the same power, the interference from $R_{2}$ to $R_{1}$ will be high and will have the same power of the transmission from $S$ to $R_{1}$. The instantaneous SINR (Signal-to-Interference-plus-Noise Ratio) in the $S-R_{1}$ link would be:

$$
S I N R_{S R_{1}}=\frac{\left|h_{S R_{1}}\right|^{2} \cdot P_{M H}}{\left|h_{R_{2} R_{1}}\right|^{2} \cdot P_{M H}+N}
$$

Thus, the co-channel interference does not allow the nodes to communicate properly. Directional antennas and interference cancellation could solve that issue, but both do not seem much realistic in a WSN scenario. Anyway, let us suppose perfect interference cancellation, which can be obtained by a combination of dirty paper and superposition coding as in [22]. Then, since simultaneous transmissions will be possible, $S$ will be able to transmit a message at alternating time slots for any number of relays, thus $L=2 \forall K$ in $\mathrm{MH}$. For instance, in the case of $K=2$, then $S I N R_{S R_{1}}=\frac{\left|h_{S R_{1}}\right|^{2} \cdot P_{M H}}{N}$, since the interference is perfectly canceled. In Section 4 we investigate the impact of interference cancellation and spatial reuse in the performance of $\mathrm{MH}$.

\subsection{Cooperative Transmission (CT)}

In the CT scheme a relay node helps the communication between $S$ and $D$. The communication happens in a two step process. In the first step $S$ broadcasts a message which is heard by both $D$ and $R_{k}$. In the second step $R_{k}$ can cooperate forwarding the message to $D$. The CT scheme requires two time slots to perform the communication and the spectral efficiency of each of its transmissions needs to be twice $(L=2)$ the spectral efficiency of SH.

Supposing the use of selection combining at the destination, the outage probability for the CT scenario is a function of the outage probabilities of each of its links $S-D, S-R_{k}$ and $R_{k}-D$ as expressed by:

$$
\mathcal{O}_{C T}=p_{S D} \cdot\left[p_{S R_{k}}+\left(1-p_{S R_{k}}\right) \cdot p_{R_{k} D}\right]
$$

or alternatively rewritten as:

$$
\mathcal{O}_{C T}=\frac{f_{S D}}{\left(P_{C T}\right)^{m}} \cdot\left[\frac{f_{S R_{k}}}{\left(P_{C T}\right)^{m}}+\left(1-\frac{f_{S R_{k}}}{\left(P_{C T}\right)^{m}}\right) \cdot \frac{f_{R_{k} D}}{\left(P_{C T}\right)^{m}}\right]
$$

where $f_{S D}=\frac{1}{\Gamma(m+1)}\left(\frac{m N \beta_{L}}{\gamma_{S D}}\right)^{m}, f_{S R_{k}}=\frac{1}{\Gamma(m+1)}\left(\frac{m N \beta_{L}}{\gamma_{S R_{k}}}\right)^{m}$ and $f_{R_{k} D}=$ $\frac{1}{\Gamma(m+1)}\left(\frac{m N \beta_{L}}{\gamma_{R_{k}} D}\right)^{m}$. Replacing $\mathcal{O}_{C T}$ by $\mathcal{O}^{*}$ in (18), the optimal transmit power $P_{C T}^{*}$ can be obtained as the smallest real and positive solution of:

$$
\mathcal{O}^{*}\left(P_{C T}\right)^{3 m}-\left(f_{S R_{k}} f_{S D}+f_{R_{k} D} f_{S D}\right)\left(P_{C T}\right)^{m}+\left(f_{S R_{k}} f_{R_{k} D} f_{S D}\right)=0 .
$$




\subsubsection{SDF}

In SDF the relay node cooperates if it was able to correctly decode the message from $S$. The total consumed energy per bit is:

$$
\begin{aligned}
E_{b t, S D F}= & p_{S R_{k}} \times \frac{P_{P A, C T}+P_{T X}+2 P_{R X}}{L R_{b}} \\
& +\left(1-p_{S R_{k}}\right) \times \frac{2 P_{P A, C T}+2 P_{T X}+3 P_{R X}}{L R_{b}} .
\end{aligned}
$$

The first term in (20) represents the consumed energy in the case that $R_{k}$ can not decode the message from $S$ properly. The second term represents the case where $R_{k}$ is successful in decoding the message. In this second case $R_{k}$ forwards the message to $D$, reflecting in additional transmit and receive consumptions.

\subsubsection{IDF}

The availability of a feedback channel is considered in the IDF cooperative protocol. The feedback channel provides to node $D$ a mechanism to inform the relay node if it could successfully decode a message, which permits the relay to retransmit an overheard message only if required by $D$. The total consumed energy per bit is ${ }^{4}$ :

$$
\begin{aligned}
E_{b t, I D F}= & \left(1-p_{S D}\right) \times \frac{P_{P A, C T}+P_{T X}+2 P_{R X}}{L R_{b}} \\
& +p_{S D} \cdot p_{S R_{k}} \times \frac{P_{P A, C T}+P_{T X}+2 P_{R X}}{L R_{b}} \\
& +p_{S D} \cdot\left(1-p_{S R_{k}}\right) \times \frac{2 P_{P A, C T}+2 P_{T X}+3 P_{R X}}{L R_{b}} .
\end{aligned}
$$

The first term in (21) represents a successful transmission from $S$ to $D$, which does not require the cooperation of the chosen relay $R_{k}$. In the second term neither $D$ nor $R_{k}$ can decode the message, which represents a failure in the transmission process. The third term represents an unsuccessful decoding at $D$ with a correct decoding at the relay node which results in the cooperation of $R_{k}$ with an additional transmission from $R_{k}$ to $D$.

Note that in the analyzed cooperative schemes, for $K>1$ we have a multirelay scenario which the optimal relay is the one that minimizes (20) and (21). We consider that the relay has been previously defined by a proactive relay selection algorithm. A proactive algorithm allows all the other nodes (except $S, D$, and the selected relay) to enter an idle mode during the twohop cooperative transmission [5].

\footnotetext{
${ }^{4}$ When a return channel is available we also consider that two time slots are reserved for the transmission of each packet. Therefore, in order to achieve the same end-to-end throughput as SH, in IDF $L=2$. If an ACK signal is received, and a retransmission is not required, then both the source and the relay stay silent during the second time slot, which impacts positively in the energy consumption [7].
} 
3.4 Hybrid Transmission (HT)

Hybrid schemes that combine the use of cooperative and non-cooperative techniques can be defined. Considering scenarios with several nodes, the number of possible combinations is very large. In order to compare the energy efficiency of some hybrid transmissions to cooperative and non-cooperative techniques, we considered scenarios with $K=2$ relays, which is the minimum number of relay nodes required to generate hybrid schemes. We detail in this section the hybrid scheme that among all the analyzed combinations presented the best performance.

Figure 1 shows the hybrid transmission scheme that combines a $\mathrm{MH}$ transmission through $R_{1}$ with cooperation through $R_{2}$. In the first time slot, $S$ transmits a frame to $R_{1}$. The transmission can be overheard by $R_{2}$ and $D$, as shown in Figure 1a. If the transmission is successfully overheard by $R_{2}$, this relay retransmits the frame to $D$ in the second time slot, even if $R_{1}$ could decode the message, as shown in Figure 1b. Note that while $R_{2}$ transmits to $D, R_{1}$ is silent. However, if $R_{2}$ can not decode the message from $S$ but $R_{1}$ could, then $R_{1}$ retransmits the frame to $D$ in the second time slot, as shown in Figure 1c. If neither $D$ nor $R_{1}$ nor $R_{2}$ can decode the message from $S$, the frame is considered lost. In order to avoid a collision in the second time slot we assume that $R_{2}$ always waits for a shorter time than $R_{1}$ before sensing the medium. Therefore, $R_{1}$ defers its transmission if a transmission from $R_{2}$ is detected. Note that in this scheme we have a mechanism similar to relay selection, where only one out of two relays cooperates with the transmission. Moreover, the communication process is defined in two time slots and the spectral efficiency of each transmission needs to be twice $(L=2)$ the spectral efficiency of $\mathrm{SH}$ in order to guarantee at least the same end-to-end throughput. This scheme can operate under both SDF and IDF protocols. The outage probability for the HT proposed scheme is given by:

$$
\begin{aligned}
\mathcal{O}_{H T}= & p_{S D} \cdot p_{S R_{1}} \cdot p_{S R_{2}}+p_{S D} \cdot\left(1-p_{S R_{2}}\right) \cdot p_{R_{2} D} \\
& +p_{S D} \cdot\left(1-p_{S R_{1}}\right) \cdot p_{S R_{2}} \cdot p_{R_{1} D},
\end{aligned}
$$

which can be rewritten as:

$$
\begin{aligned}
\mathcal{O}_{H T}= & \frac{f_{S D} \cdot f_{S R_{1}} \cdot f_{S R_{2}}}{\left(P_{H T}\right)^{3 m}}+\left(1-\frac{f_{S R_{2}}}{\left(P_{H T}\right)^{m}}\right) \cdot \frac{f_{S D} \cdot f_{R_{2} D}}{\left(P_{H T}\right)^{2 m}} \\
& +\left(1-\frac{k_{S R_{1}}}{\left(P_{H T}\right)^{m}}\right) \cdot \frac{f_{S D} \cdot f_{S R_{2}} \cdot f_{R_{1} D}}{\left(P_{H T}\right)^{3 m}}
\end{aligned}
$$

Replacing $\mathcal{O}_{H T}$ by $\mathcal{O}^{*}$ in $(23)$, the optimal transmit power $P_{H T}^{*}$ can be obtained as the smallest real and positive solution of:

$$
\begin{aligned}
& \mathcal{O}^{*}\left(P_{H T}\right)^{4 m}-\left(f_{S D} f_{R_{2} D}\right)\left(P_{H T}\right)^{2 m} \\
& -\left(f_{S D} f_{S R_{1}} f_{S R_{2}}+f_{S D} f_{S R_{2}} f_{R_{1} D}-f_{S D} f_{S R_{2}} f_{R_{2} D}\right)\left(P_{H T}\right)^{m}+ \\
& +\left(f_{S D} f_{S R_{1}} f_{S R_{2}} f_{R_{1} D}\right)=0 .
\end{aligned}
$$


3.4.1 Selective Hybrid Transmission (SHT)

In the HT scheme using the SDF protocol, as a feedback channel is not available, the decoded message is always retransmitted by one relay node, be it $R_{1}$ or $R_{2}$, unless both could not decode the source message. The total consumed energy per bit is:

$$
\begin{aligned}
E_{b t, S H T}= & p_{S R_{1}} \cdot p_{S R_{2}} \times \frac{P_{P A, H T}+P_{T X}+3 P_{R X}}{L R_{b}} \\
& +\left(1-p_{S R_{2}}\right) \times \frac{2 P_{P A, H T}+2 P_{T X}+4 P_{R X}}{L R_{b}} \\
& +\left(1-p_{S R_{1}}\right) \cdot p_{S R_{2}} \times \frac{2 P_{P A, H T}+2 P_{T X}+4 P_{R X}}{L R_{b}} .
\end{aligned}
$$

The first term in (25) represents the consumed energy in the case that neither $R_{1}$ nor $R_{2}$ can decode the message from $S$ properly. The second term represents the case where $R_{2}$ is successful in decoding the message and forwards the frame to $D$. The third term represents the case that only $R_{1}$ can decode the transmission from $S$ and then forwards the frame to $D$.

\subsubsection{Incremental Hybrid Transmission (IHT)}

The presence of a feedback channel in the IHT scheme provides a mechanism so that $D$ requests retransmissions from the relay nodes only if needed. The total consumed energy per bit in this case is:

$$
\begin{aligned}
E_{b t, I H T}= & p_{S R_{1}} \cdot p_{S R_{2}} \times \frac{P_{P A, H T}+P_{T X}+3 P_{R X}}{L R_{b}} \\
& +\left(1-p_{S D}\right) \cdot\left(1-p_{S R_{2}}\right) \times \frac{P_{P A, H T}+P_{T X}+3 P_{R X}}{L R_{b}} \\
& +p_{S D} \cdot\left(1-p_{S R_{2}}\right) \times \frac{2 P_{P A, H T}+2 P_{T X}+4 P_{R X}}{L R_{b}} \\
& +\left(1-p_{S D}\right) \cdot\left(1-p_{S R_{1}}\right) \cdot p_{S R_{2}} \times \frac{P_{P A, H T}+P_{T X}+3 P_{R X}}{L R_{b}} \\
& +p_{S D} \cdot\left(1-p_{S R_{1}}\right) \cdot p_{S R_{2}} \times \frac{2 P_{P A, H T_{1}}+2 P_{T X}+4 P_{R X}}{L R_{b}} .
\end{aligned}
$$

The first term in (26) represents the consumed energy in the case that neither $R_{1}$ nor $R_{2}$ can decode the message from $S$ properly. The second and third terms represent the cases where $R_{2}$ is successful in decoding the transmission from $S$. In the second term $D$ is also able to decode the message, thus no retransmission is required. In the third term, as $D$ can not decode the frame, $R_{2}$ cooperates with an additional transmission. In the forth term $R_{1}$ decodes the transmission, while $R_{2}$ can not, but the frame is also decoded by $D$ and a retransmission is not needed. Finally, the fifth term represents the case where $R_{1}$ is the only node that can decode the transmission from $S$ and retransmits the message to $D$. 
4 Results and analysis

In this section the energy efficiency of the cooperative, non-cooperative and hybrid schemes are numerically evaluated. The system parameters were set according to Table 1 and the circuitry consumption parameters follow the values presented in [10], and are listed in Table 2. We considered 500 topologies in which the relays were randomly disposed, and evaluated the mean consumed energy per bit for each transmission scheme. Figure 2 compares the mean consumed energy per bit in NLOS condition for SH, MH, SDF and IDF cooperative schemes, and the SHT and IHT hybrid schemes. It is considered for all methods a maximum outage of $\mathcal{O}^{*}=10^{-3}$ and $\Delta=2 \mathrm{~b} / \mathrm{s} / \mathrm{Hz}$. From the figure we can see that the $\mathrm{MH}$ scheme presents a worse performance even than $\mathrm{SH}$ (MH is the multi-hop transmission composed of three hops: from $S$ to $R_{1}$, then $R_{1}$ to $R_{2}$ and finally $R_{2}$ to $D$, thus $K=2$, and $\left.\mathcal{A}(w)=[1,2]\right)$. The two cooperative schemes, SDF and IDF, are outperformed by their respective HT schemes from a given distance. The SHT scheme presents a better performance than SDF for $d>14 \mathrm{~m}$. If a feedback channel is available, IHT outperforms IDF for $d>16 \mathrm{~m}$. The HT schemes outperform the CT models due to the increased spatial diversity that they provide. For example, the CT schemes provide two possible independent paths towards $D$ (from $S$ to $D$ and from $R_{k}$ to $D$ ), while in the analyzed HT models, as we have an additional relay that can cooperate with the transmission, there are three possible independent paths to $D$. For shorter distances, as the circuitry consumption is a relevant factor, the HT schemes are outperformed by the CT models due to the additional node employed in the communication. Note also that for $d>33$ $\mathrm{m}$ SHT has a better performance even than the IDF scheme, showing that the spatial diversity provided by the additional relay can be more interesting than the use of a feedback channel.

Figure 3 illustrates the mean consumed energy per bit for the same scenario as Figure 2, but considering some LOS. Although in LOS conditions the use of cooperation provides decreased overall advantage over the other noncooperative schemes, IDF and IHT still present the best performances, with IHT outperforming IDF for $d>96 \mathrm{~m}$. Moreover, SDF is outperformed by SH for $d<60 \mathrm{~m}$ and by SHT for $d>71 \mathrm{~m}$. Similarly to the results obtained for NLOS conditions, $\mathrm{SH}$ outperforms the $\mathrm{MH}$ scheme.

\subsection{Impact of throughput and outage probability}

Different results can be obtained if other values are considered for the end-toend throughput. Figure 4 shows the cooperative, non-cooperative and hybrid schemes mean consumed energy for $\Delta=6 \mathrm{~b} / \mathrm{s} / \mathrm{Hz}$ and $\mathcal{O}^{*}=10^{-3}$ in NLOS conditions, where SDF is outperformed by SH for any $d$. The results for LOS for this same scenario are shown in Figure 5. Note that $\mathrm{SH}$ outperforms all the other schemes for almost any $d$. In Figure 6 the mean energy consumption of the transmission schemes in LOS are compared for $\Delta$ ranging from $1 \mathrm{~b} / \mathrm{s} / \mathrm{Hz}$ 
to $10 \mathrm{~b} / \mathrm{s} / \mathrm{Hz}$ and for a given $S-D$ distance $(d=40 \mathrm{~m})$. Note that for $\Delta=5 \mathrm{~b} / \mathrm{s} / \mathrm{Hz}, \mathrm{SH}$ and IHT present a very close performance. However, for $\Delta>5 \mathrm{~b} / \mathrm{s} / \mathrm{Hz}, \mathrm{SH}$ outperforms the other methods. Under NLOS conditions, the advantage of $\mathrm{SH}$ decreases and it is the most energy efficient scheme only for $\Delta>8 \mathrm{~b} / \mathrm{s} / \mathrm{Hz}$, as shown in Figure 7 . Moreover, several different scenarios for other $\mathcal{O}^{*}$ (ranging from $10^{-2}$ to $10^{-4}$ ) were tested, without relevant changes in the conclusions.

\subsection{Spatial reuse in $\mathrm{MH}$}

Figure 8 shows that as the number of available relays $(K)$ in $\mathrm{MH}$ is increased, the mean energy consumption also increases. That is because although a higher number of relays allows shorter hops, the spectral efficiency to be used in each hop also increases, as the maximum imposed delay for $\mathrm{MH}$ is $K+1$ times that of SH. However, different results can be obtained if spatial reuse is considered. In Figure 9 we show the energy efficiency of $\mathrm{MH}$ under $\mathrm{LOS}, \mathcal{O}^{*}=10^{-3}$ and $\Delta=2 \mathrm{~b} / \mathrm{s} / \mathrm{Hz}$, when spatial reuse is applied, and the interference is optimally canceled. Note that with spatial reuse $\mathrm{MH}$ with $K=1$ is the most energy efficient $\mathrm{MH}$ scheme for short distances. However, for higher $S-D$ distances, the $\mathrm{MH}$ schemes with different $K$ present similar performances. That is because, although the transmit power to be used at each hop is reduced with the increase of $K$, more nodes are involved in the communication, and then the circuitry consumption becomes relevant. We can also note that with spatial reuse, $\mathrm{MH}$ is only able to outperform SDF for short $S-D$ distances, but is outperformed by $\mathrm{SH}$ for any $d$. In the case of NLOS, MH with spatial reuse is not more energy efficient than SDF or SH for any $d$. Therefore, considering our scenarios, $\mathrm{MH}$ even with spatial reuse only outperforms SDF in the case of LOS and when the $S-D$ distance is small.

\subsection{Impact of the end-to-end throughput constraints}

In this section we relax the end-to-end throughput constraints in order to show that much different results are obtained if that is not considered. Figure 10 shows the mean energy consumed by the cooperative and non-cooperative schemes with no end-to-end throughput restrictions ( $L=1$ for all schemes), in NLOS conditions. Although SDF presents the best performance for most $d$, $\mathrm{SH}$ is the most advantageous scheme at short distances. The $\mathrm{SH}$ transmission is outperformed by SDF for $d>9 \mathrm{~m}$ and by MH with $K=2$ and $K=3$ for $d>$ $16 \mathrm{~m}$. Moreover, note that in this scenario $\mathrm{MH}$ using shorter hops can be more efficient than $\mathrm{SH}$, which opposes the results presented in Figure 8, where the end-to-end throughput restrictions are considered and $\mathrm{MH}$ is actually the worst option. The opposing conclusions of Figures 10 and 8 show the importance of considering the appropriate modeling in an energy efficiency analysis. 


\section{Conclusion}

The energy efficiency of some cooperative, non-cooperative and hybrid transmission schemes in WSN scenarios composed of multiple nodes are compared in this paper. The analysis is performed under the constraint of a target outage probability and end-to-end throughput in LOS and NLOS conditions. Our analysis shows that in most scenarios cooperative and hybrid transmission schemes present the best performances, specially if a feedback channel is available. Among the non-cooperative schemes, the SH transmission presents the best results. However, in systems where the end-to-end throughput is not a requirement, different results are obtained. In this case, $\mathrm{MH}$ using several nodes can be more energy efficient than SH or SDF, due to the shortened distance of each hop. Anyway, if end-to-end throughput is a concern, then MH should be avoided and the use of cooperation, specially of the incremental type, is in general the best option. 


\section{References}

1. Akyildiz, I., Su, W., Sankarasubramaniam, Y., Cayirci, E.: A survey on sensor networks. Communications Magazine, IEEE 40(8), 102 - 114 (2002). DOI 10.1109/MCOM.2002. 1024422

2. Biglieri, E., Caire, G., Taricco, G.: Limiting performance of block-fading channels with multiple antennas. Information Theory, IEEE Transactions on 47(4), 1273-1289 (2001). DOI 10.1109/18.923715

3. Biglieri, E., Calderbank, R., Constantinides, A., Goldsmith, A., Paulraj, A., Poor, H.V.: MIMO Wireless Communications. Cambridge University Press, New York, NY, USA (2007)

4. Biglieri, E., Proakis, J., Shamai, S.: Fading channels: information-theoretic and communications aspects. Information Theory, IEEE Transactions on 44(6), 2619 -2692 (1998). DOI 10.1109/18.720551

5. Bletsas, A., Shin, H., Win, M.: Cooperative communications with outage-optimal opportunistic relaying. Wireless Communications, IEEE Transactions on 6(9), 3450 -3460 (2007). DOI 10.1109/TWC.2007.06020050

6. Bougard, B., Lenoir, G., Dejonghe, A., Van der Perre, L., Catthoor, F., Dehaene, W.: Smart mimo: an energy-aware adaptive mimo-ofdm radio link control for nextgeneration wireless local area networks. EURASIP J. Wirel. Commun. Netw. 2007, 13:1-13:12 (2007). DOI http://dx.doi.org/10.1155/2007/98186. URL http://dx.doi. org/10.1155/2007/98186

7. Brante, G., Kakitani, M., Souza, R.: Energy efficiency analysis of some cooperative and non-cooperative transmission schemes in wireless sensor networks. Communications, IEEE Transactions on PP(99), 1 -7 (2011). DOI 10.1109/TCOMM.2011.063011.100744

8. Chen, G., Hanson, S., Blaauw, D., Sylvester, D.: Circuit design advances for wireless sensing applications. Proceedings of the IEEE 98(11), $1808-1827$ (2010). DOI 10.1109/ JPROC.2010.2053333

9. Cui, S., Goldsmith, A., Bahai, A.: Energy-efficiency of mimo and cooperative mimo techniques in sensor networks. Selected Areas in Communications, IEEE Journal on 22(6), 1089 - 1098 (2004). DOI 10.1109/JSAC.2004.830916

10. Cui, S., Goldsmith, A., Bahai, A.: Energy-constrained modulation optimization. Wireless Communications, IEEE Transactions on 4(5), 2349 - 2360 (2005). DOI 10.1109/ TWC.2005.853882

11. Goldsmith, A.: Wireless Communications. Cambridge University Press, New York, NY, USA (2005)

12. Heliot, F., Imran, M.A., Tafazolli, R.: On the energy efficiency gain of mimo communication under various power consumption models. In: Future Network and Mobile Summit (2011)

13. Jacobson, K., Krzymien, W.: Cooperative vs multihop relaying in cellular systems. In: Personal, Indoor and Mobile Radio Communications, 2009 IEEE 20th International Symposium on, pp. 1853 -1857 (2009). DOI 10.1109/PIMRC.2009.5450005

14. Knopp, R., Humblet, P.: On coding for block fading channels. Information Theory, IEEE Transactions on 46(1), 189 -205 (2000). DOI 10.1109/18.817517

15. Laneman, J., Tse, D., Wornell, G.: Cooperative diversity in wireless networks: Efficient protocols and outage behavior. Information Theory, IEEE Transactions on 50(12), 3062 - 3080 (2004). DOI 10.1109/TIT.2004.838089

16. Laneman, J., Wornell, G.: Energy-efficient antenna sharing and relaying for wireless networks. In: Wireless Communications and Networking Conference, 2000. WCNC. 2000 IEEE, vol. 1, pp. 7 -12 vol.1 (2000). DOI 10.1109/WCNC.2000.904590

17. Liu, X., Goldsmith, A.: Wireless network design for distributed control. In: Decision and Control, 2004. CDC. 43rd IEEE Conference on, vol. 3, pp. $2823-2829$ Vol.3 (2004). DOI 10.1109/CDC.2004.1428892

18. Lo, C., Vishwanath, S., Heath, R.: An energy-based comparison of long-hop and shorthop routing in mimo networks. Vehicular Technology, IEEE Transactions on 59(1), 394 -405 (2010). DOI 10.1109/TVT.2009.2033075

19. Malkamaki, E., Leib, H.: Coded diversity on block-fading channels. Information Theory, IEEE Transactions on 45(2), $771-781$ (1999). DOI 10.1109/18.749028 
20. Ong, L., Motani, M.: Optimal routing for decode-forward in cooperative wireless networks. Communications, IEEE Transactions on 58(8), $2345-2355$ (2010). DOI 10.1109/TCOMM.2010.08.090134

21. Paulraj, A., Nabar, R., Gore, D.: Introduction to Space-Time Wireless Communications. Cambridge University Press, New York, NY, USA (2003)

22. Pereira, R., Souza, R., Pellenz, M.: Multiple concurrent transmissions in wireless mesh networks employing superposition and dirty paper coding. Vehicular Technology, IEEE Transactions on 58(9), $5115-5123$ (2009). DOI 10.1109/TVT.2009.2023792

23. Qi, Y., Imran, M., Hoshyar, R.: H2-arq relaying: Spectrum and energy efficiency analysis. submitted to IEEE Journal on Selected Areas in Communications 2011 -, - (2011)

24. Sadek, A.K., Yu, W., Liu, K.J.R.: On the energy efficiency of cooperative communications in wireless sensor networks. ACM Trans. Sen. Netw. 6(1), 1-21 (2009). DOI http://doi.acm.org/10.1145/1653760.1653765

25. Savazzi, S., Spagnolini, U.: Cooperative space-time coded transmissions in nakagami-m fading channels. In: Global Telecommunications Conference, 2007. GLOBECOM '07. IEEE, pp. $4334-4338$ (2007). DOI 10.1109/GLOCOM.2007.824

26. Sendonaris, A., Erkip, E., Aazhang, B.: User cooperation diversity. part i. system description. Communications, IEEE Transactions on 51(11), 1927 - 1938 (2003). DOI 10.1109/TCOMM.2003.818096

27. Siam, M., Krunz, M., Cui, S., Muqattash, A.: Energy-efficient protocols for wireless networks with adaptive mimo capabilities. Wireless Networks 16, 199-212 (2010). URL http://dx.doi.org/10.1007/s11276-008-0124-3. 10.1007/s11276-008-0124-3

28. Simon, M.K., Alouini, M.S.: Digital Communication over Fading Channels. Wiley Interscience (2004)

29. Wang, Z., Giannakis, G.: A simple and general parameterization quantifying performance in fading channels. IEEE Transac. on Commun. 51(8), 1389 - 1398 (2003). DOI 10.1109/TCOMM.2003.815053

30. Xiong, Z., Liveris, A., Cheng, S.: Distributed source coding for sensor networks. Signal Processing Magazine, IEEE 21(5), 80 - 94 (2004). DOI 10.1109/MSP.2004.1328091

31. Yacoub, M.: The $\kappa-\mu$ distribution and the $\eta-\mu$ distribution. Antennas and Propagation Magazine, IEEE 49(1), 68 -81 (2007). DOI 10.1109/MAP.2007.370983

32. Zhou, Z. Zhou, S. Cui, J.-H, Cui, S.: Energy-efficient cooperative communication based on power control and selective single-relay in wireless sensor networks. IEEE Transac. Wireless Commun. 7(8), 3066-3078 (2008). DOI 10.1109/TWC.2008.061097 
List of Tables

1 System Parameters . . . . . . . . . . . . . . . . 20

2 Circuitry Power Consumption . . . . . . . . . . . . . . . 21 
Table 1 System Parameters

\begin{tabular}{l|l}
\hline Link Margin & $M_{l}=40 \mathrm{~dB}$ \\
Noise Figure & $N_{f}=10 \mathrm{~dB}$ \\
Antenna Gain & $G=5 \mathrm{dBi}$ \\
Carrier Frequency & $f_{c}=2.5 \mathrm{GHz}$ \\
Noise Power Spectral Density & $N_{0}=-174 \mathrm{dBm}$ \\
Bandwidth & $B=10 \mathrm{KHz}$ \\
Path Loss Exponent (LOS) & $\alpha=2.5$ \\
Path Loss Exponent (NLOS) & $\alpha=3.5$ \\
\hline
\end{tabular}


Table 2 Circuitry Power Consumption

\begin{tabular}{l|l}
\hline Mixer & $P_{\operatorname{mix}}=30.0 \mathrm{~mW}$ \\
TX/RX Filters & $P_{f i l_{-} t x}=P_{f i l \_r x}=2.5 \mathrm{~mW}$ \\
Frequency Synthesizer & $P_{s y n}=50 \mathrm{~mW}$ \\
Low-Noise Amplifier & $P_{L N A}=20 \mathrm{~mW}$ \\
Intermediate Frequency Amplifier & $P_{I F A}=3 \mathrm{~mW}$ \\
Analog-to-Digital Converter & $P_{A D C}=6.7 \mathrm{~mW}$ \\
Digital-to-Analog Converter & $P_{D A C}=15.4 \mathrm{~mW}$ \\
Amplifier Drain Efficiency & $\eta=0.35$ \\
\hline
\end{tabular}




\section{List of Figures}

1 Hybrid transmission scheme. . . . . . . . . . . . . . . . . 23

2 Mean consumed energy per bit of the Single-hop, Multi-hop through $R_{1}$ and $R_{2}$, Cooperative, and Hybrid schemes in NLOS condition, for $K=2, \mathcal{O}^{*}=10^{-3}$ and $\Delta=2 \mathrm{~b} / \mathrm{s} / \mathrm{Hz}$. . . . . . 24

3 Mean consumed energy per bit of the Single-hop, Multi-hop through $R_{1}$ and $R_{2}$, Cooperative, and Hybrid schemes in LOS condition, for $K=2, \mathcal{O}^{*}=10^{-3}$ and $\Delta=2 \mathrm{~b} / \mathrm{s} / \mathrm{Hz}$. . . . . .

4 Mean consumed energy per bit of the Single-hop, Multi-hop through $R_{1}$ and $R_{2}$, Cooperative, and Hybrid schemes in NLOS condition, for $K=2, \mathcal{O}^{*}=10^{-3}$ and $\Delta=6 \mathrm{~b} / \mathrm{s} / \mathrm{Hz} . \ldots . .26$

5 Mean consumed energy per bit of the Single-hop, Multi-hop through $R_{1}$ and $R_{2}$, Cooperative, and Hybrid schemes in LOS condition, for $K=2, \mathcal{O}^{*}=10^{-3}$ and $\Delta=6 \mathrm{~b} / \mathrm{s} / \mathrm{Hz}$. . . . . . 27

6 Mean consumed energy per bit of the Single-hop, Multi-hop through $R_{1}$ and $R_{2}$, Cooperative, and Hybrid schemes in LOS condition, for $K=2, \mathcal{O}^{*}=10^{-3}$ and $d=40 \mathrm{~m}$. . . . . . . 28

7 Mean consumed energy per bit of the Single-hop, Multi-hop through $R_{1}$ and $R_{2}$, Cooperative, and Hybrid schemes in NLOS condition, for $K=2, \mathcal{O}^{*}=10^{-3}$ and $d=40 \mathrm{~m}$. . . . . . . .

8 Mean consumed energy per bit of the Single-hop, Multi-hop and SDF, considering the end-to-end throughput restrictions in NLOS condition, for $\mathcal{O}^{*}=10^{-3}$ and $\Delta=2 \mathrm{~b} / \mathrm{s} / \mathrm{Hz}$. . . . . . .

9 Mean consumed energy per bit of the Single-hop, Multi-hop and SDF, in LOS condition for $\mathcal{O}^{*}=10^{-3}$ and $\Delta=2 \mathrm{~b} / \mathrm{s} / \mathrm{Hz}$. Spatial reuse is considered for Multi-hop. . . . . . . . . .

10 Total consumed energy per bit of the Single-hop, Multi-hop and SDF, with no end-to-end throughput constraints $(L=1)$ in NLOS condition, for $\mathcal{O}^{*}=10^{-3}$ and $\Delta=2 \mathrm{~b} / \mathrm{s} / \mathrm{Hz}$. . . . . 32 
Main Transmission

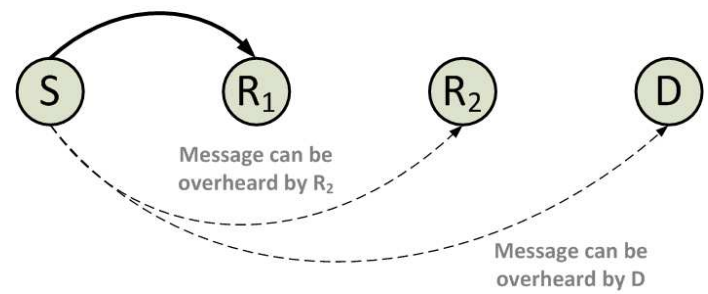

(a) The transmission from node $S$ to $R_{1}$ can be overheard by $R_{2}$ and $D$.

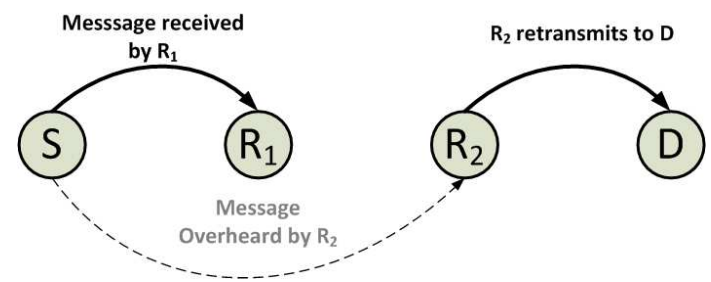

(b) Node $R_{2}$ overheard the transmission from $S$ and retransmitted the frame to $D$.

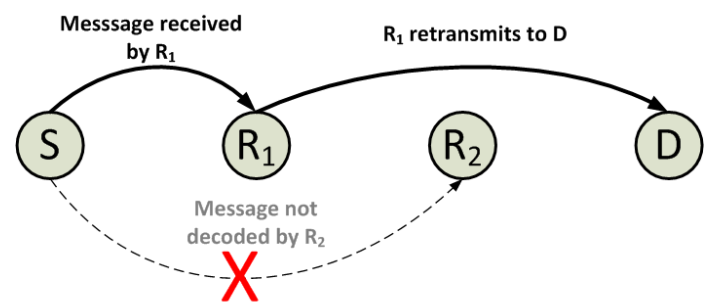

(c) Node $R_{2}$ could not successfully overhear the transmission from $S$, then $R_{1}$ retransmitted the frame to $D$.

Fig. 1 Hybrid transmission scheme. 


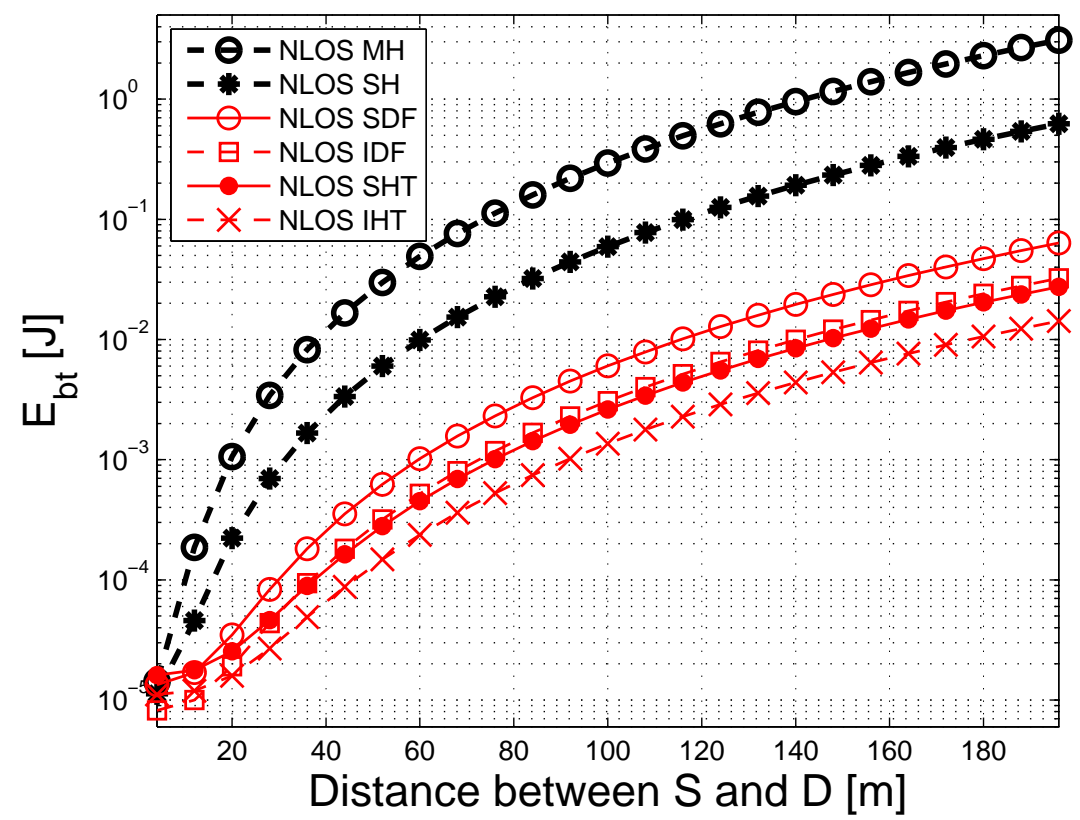

Fig. 2 Mean consumed energy per bit of the Single-hop, Multi-hop through $R_{1}$ and $R_{2}$, Cooperative, and Hybrid schemes in NLOS condition, for $K=2, \mathcal{O}^{*}=10^{-3}$ and $\Delta=2$ $\mathrm{b} / \mathrm{s} / \mathrm{Hz}$. 


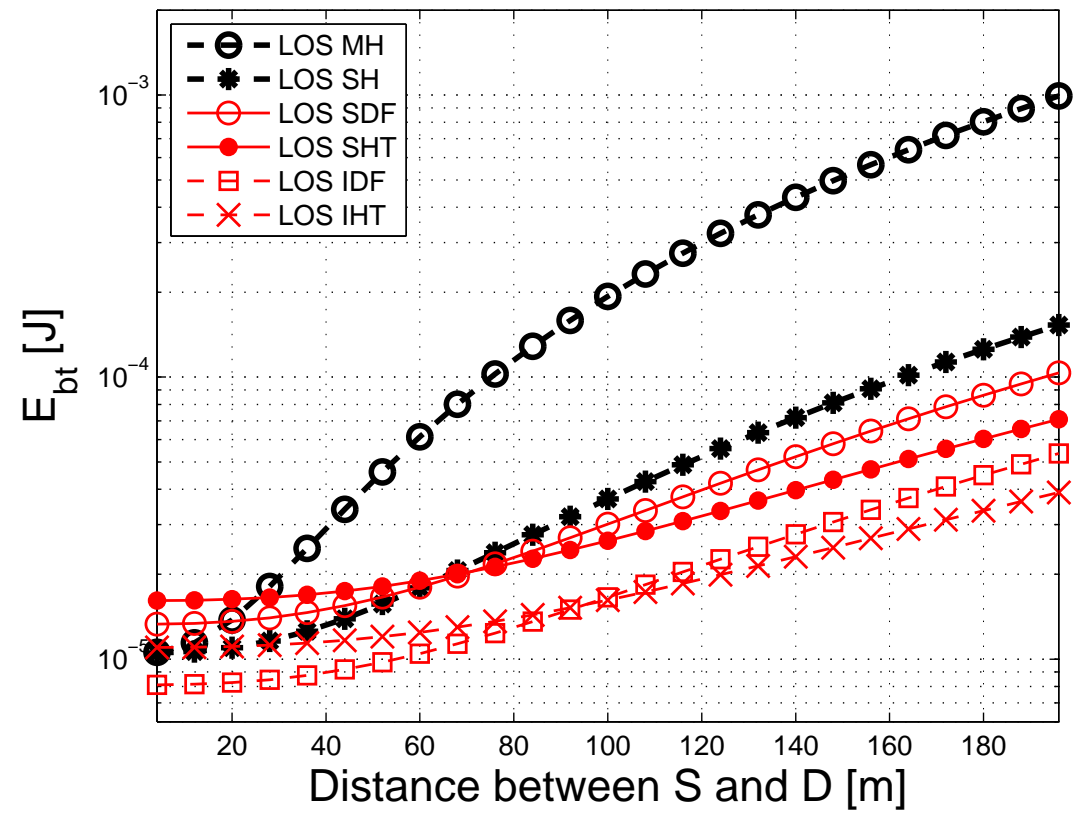

Fig. 3 Mean consumed energy per bit of the Single-hop, Multi-hop through $R_{1}$ and $R_{2}$, Cooperative, and Hybrid schemes in LOS condition, for $K=2, \mathcal{O}^{*}=10^{-3}$ and $\Delta=2$ $\mathrm{b} / \mathrm{s} / \mathrm{Hz}$. 


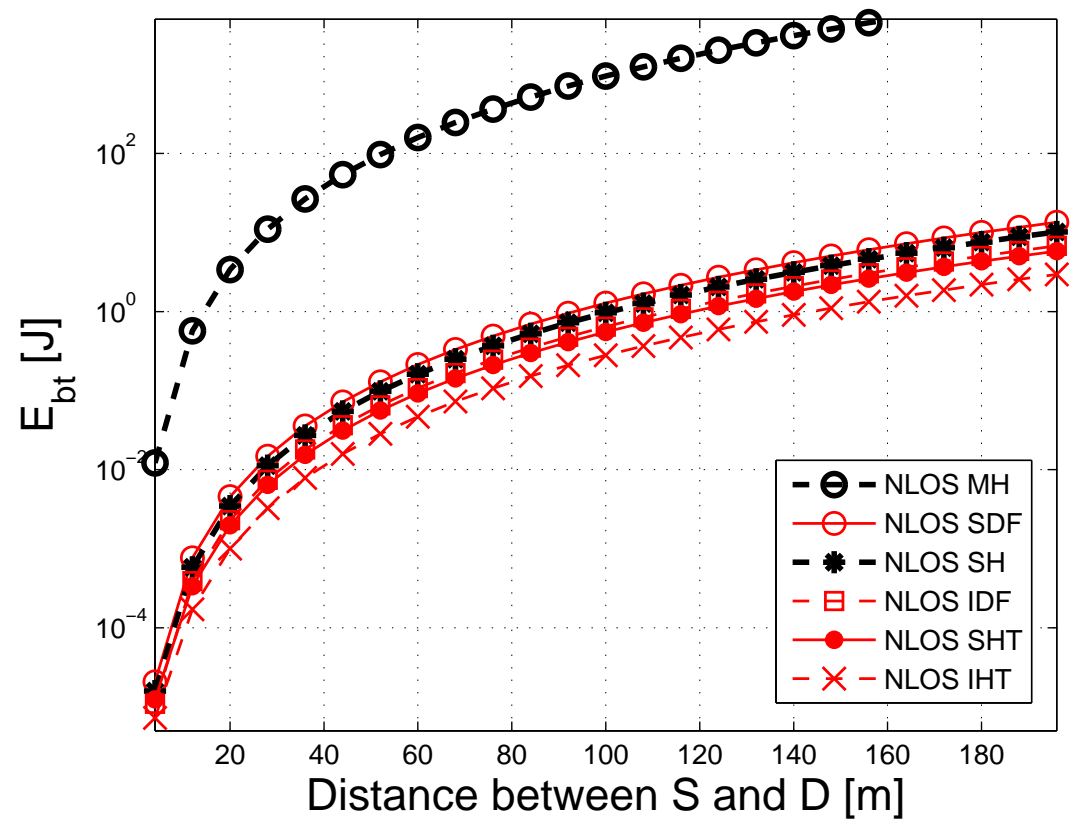

Fig. 4 Mean consumed energy per bit of the Single-hop, Multi-hop through $R_{1}$ and $R_{2}$, Cooperative, and Hybrid schemes in NLOS condition, for $K=2, \mathcal{O}^{*}=10^{-3}$ and $\Delta=6$ $\mathrm{b} / \mathrm{s} / \mathrm{Hz}$. 


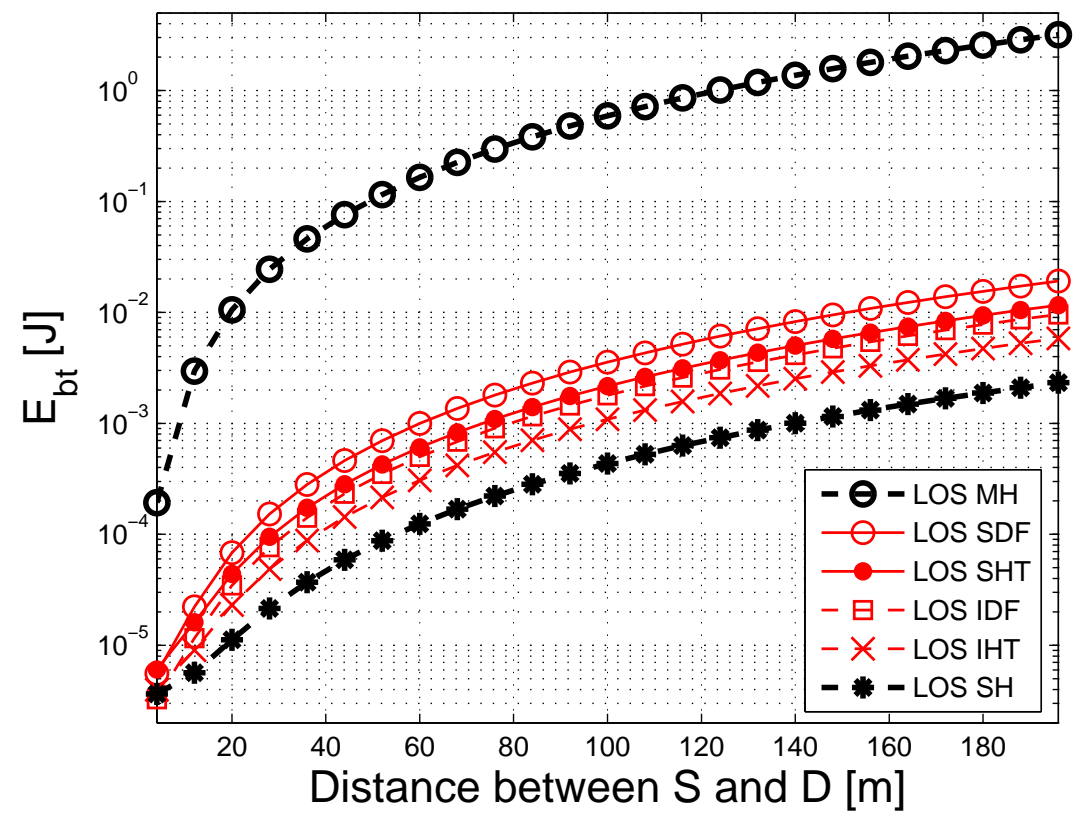

Fig. 5 Mean consumed energy per bit of the Single-hop, Multi-hop through $R_{1}$ and $R_{2}$, Cooperative, and Hybrid schemes in LOS condition, for $K=2, \mathcal{O}^{*}=10^{-3}$ and $\Delta=6$ $\mathrm{b} / \mathrm{s} / \mathrm{Hz}$. 


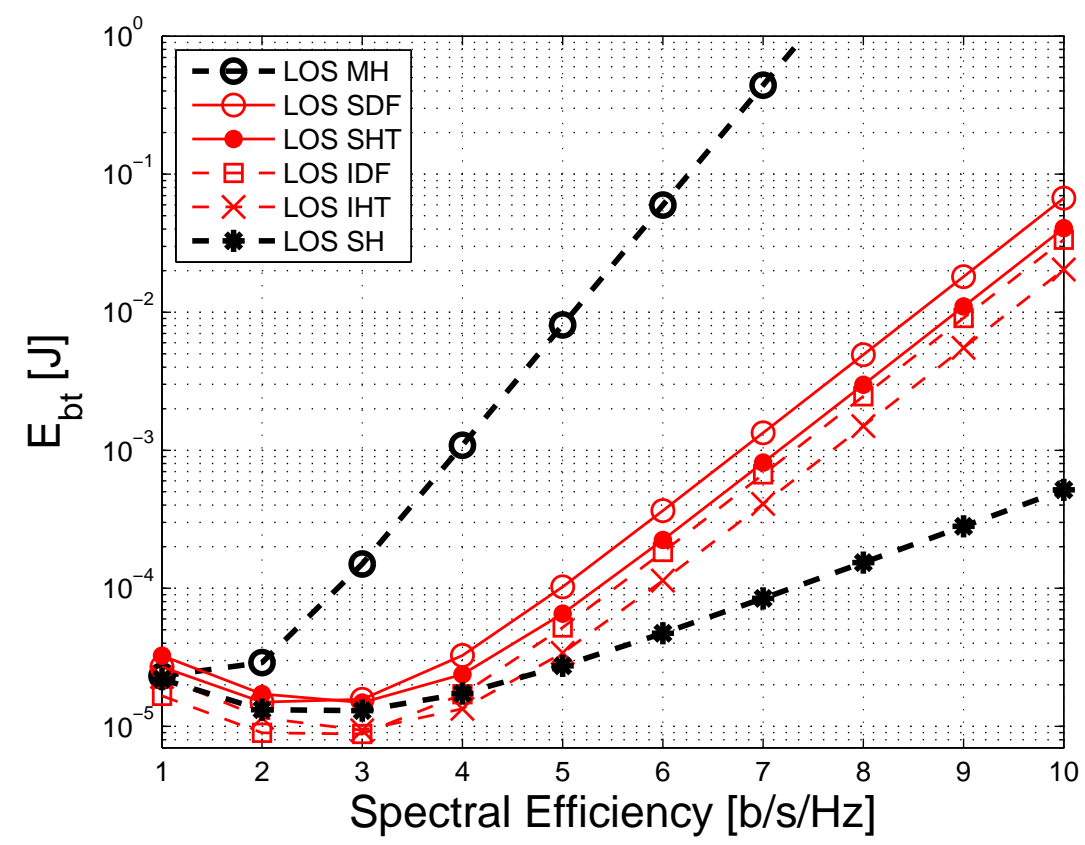

Fig. 6 Mean consumed energy per bit of the Single-hop, Multi-hop through $R_{1}$ and $R_{2}$, Cooperative, and Hybrid schemes in LOS condition, for $K=2, \mathcal{O}^{*}=10^{-3}$ and $d=40 \mathrm{~m}$. 


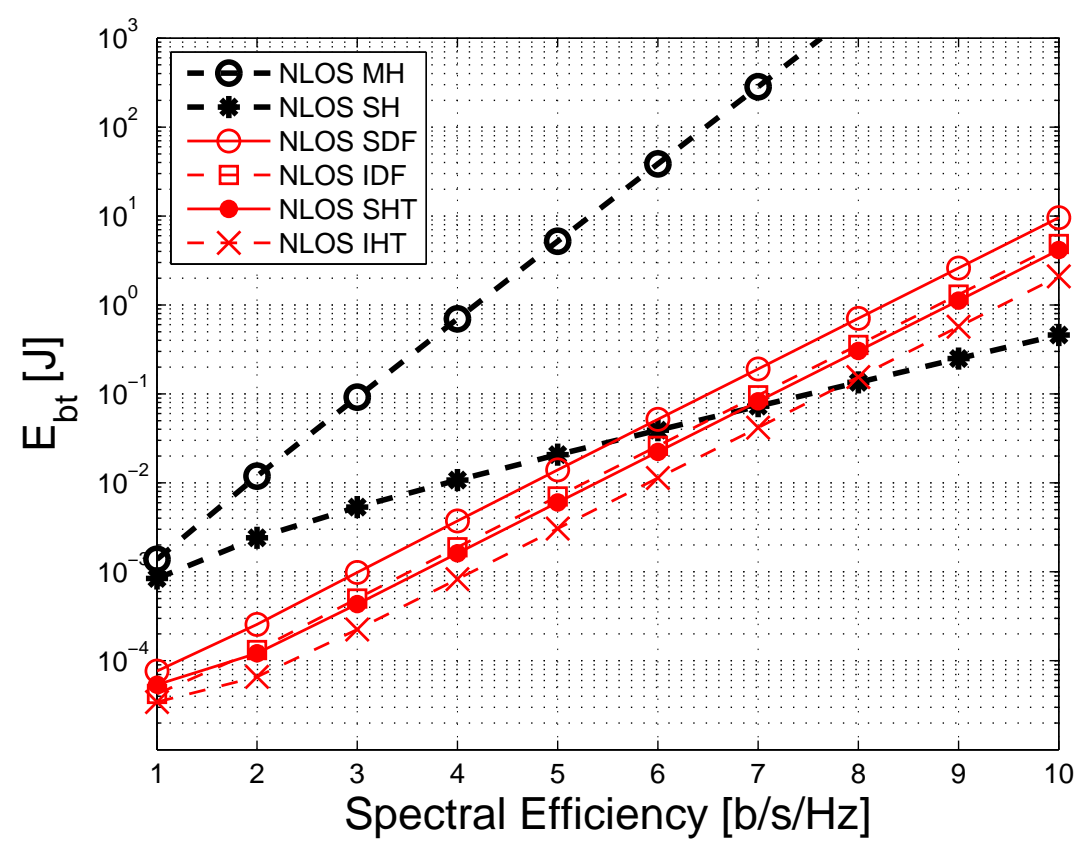

Fig. 7 Mean consumed energy per bit of the Single-hop, Multi-hop through $R_{1}$ and $R_{2}$, Cooperative, and Hybrid schemes in NLOS condition, for $K=2, \mathcal{O}^{*}=10^{-3}$ and $d=40 \mathrm{~m}$. 


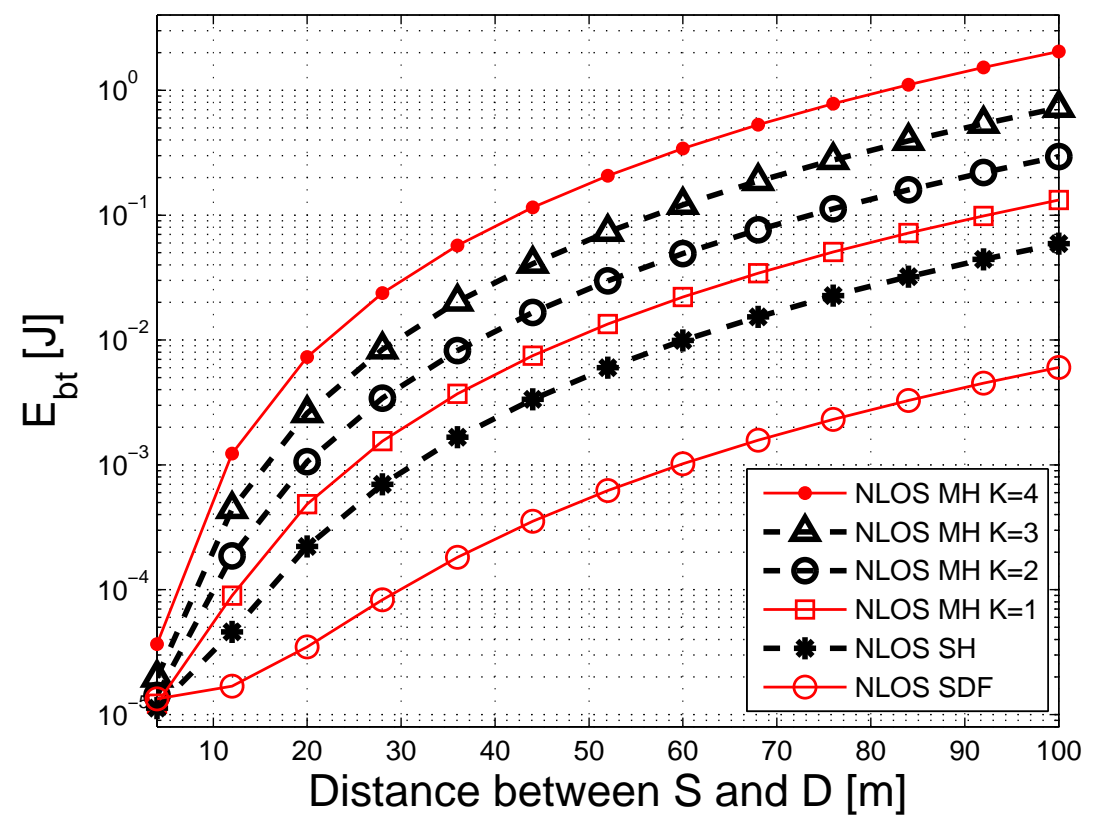

Fig. 8 Mean consumed energy per bit of the Single-hop, Multi-hop and SDF, considering the end-to-end throughput restrictions in NLOS condition, for $\mathcal{O}^{*}=10^{-3}$ and $\Delta=2$ $\mathrm{b} / \mathrm{s} / \mathrm{Hz}$. 


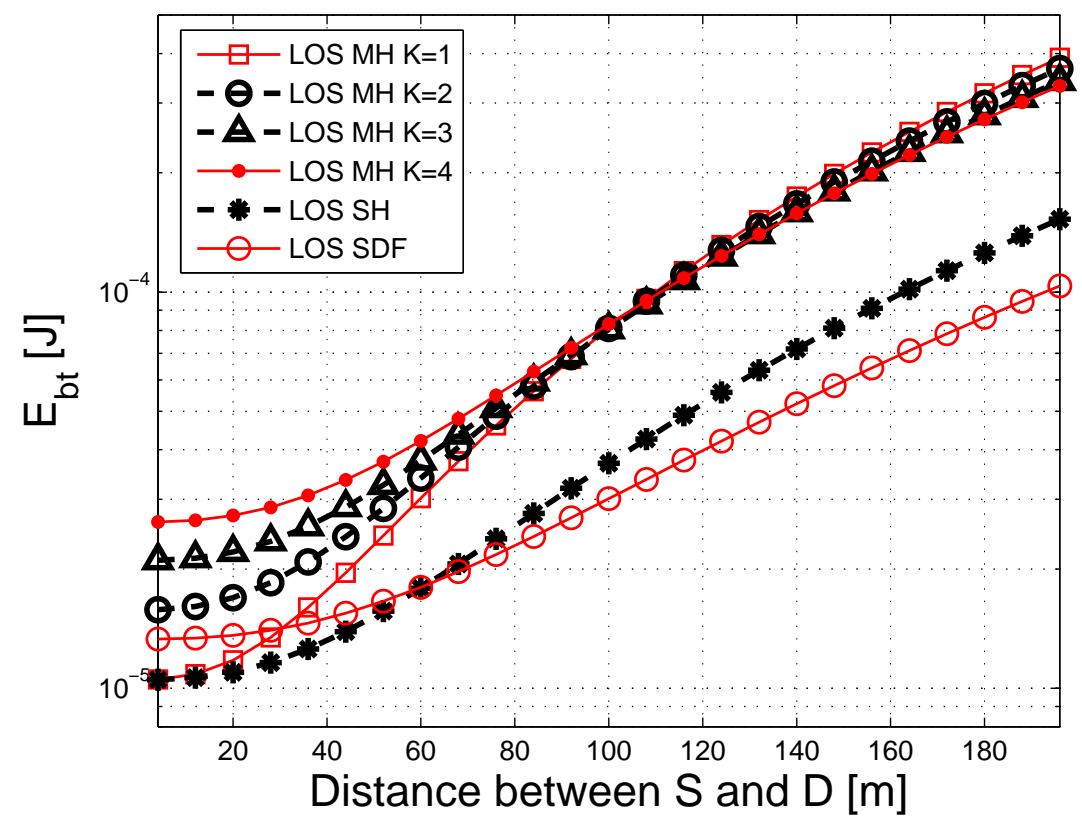

Fig. 9 Mean consumed energy per bit of the Single-hop, Multi-hop and SDF, in LOS condition for $\mathcal{O}^{*}=10^{-3}$ and $\Delta=2 \mathrm{~b} / \mathrm{s} / \mathrm{Hz}$. Spatial reuse is considered for Multi-hop. 


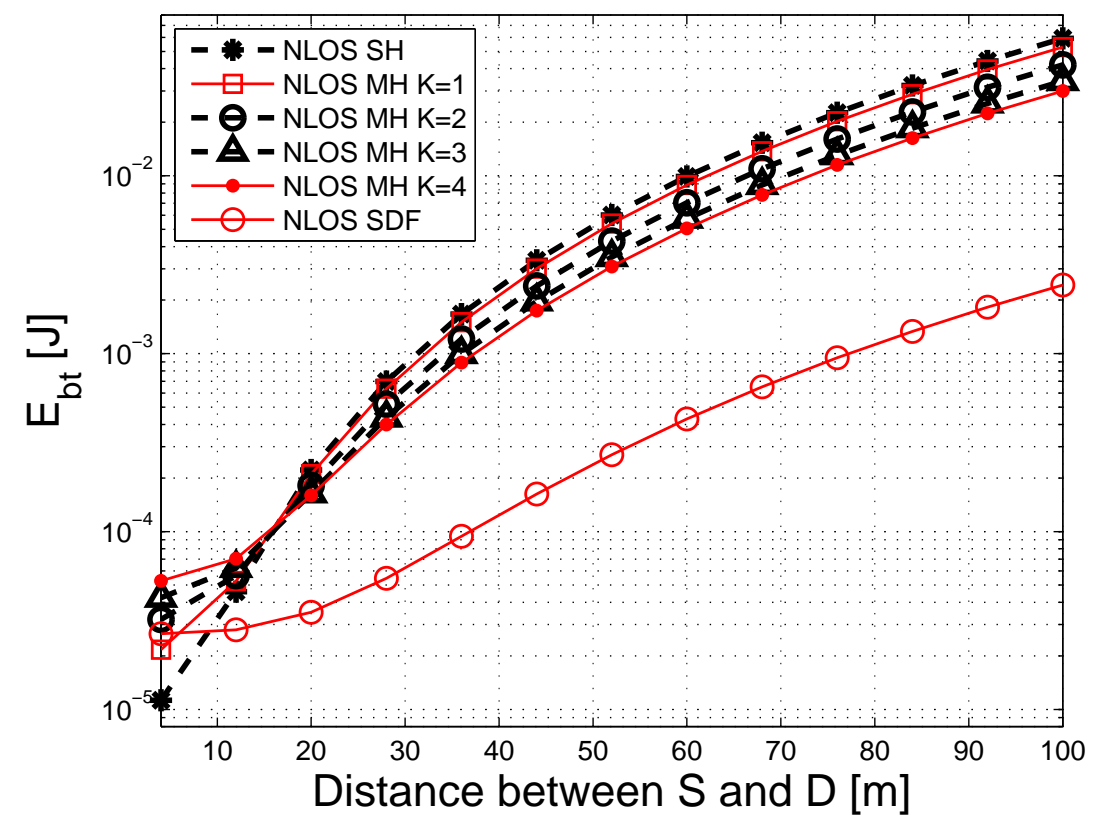

Fig. 10 Total consumed energy per bit of the Single-hop, Multi-hop and SDF, with no end-to-end throughput constraints $(L=1)$ in NLOS condition, for $\mathcal{O}^{*}=10^{-3}$ and $\Delta=2$ $\mathrm{b} / \mathrm{s} / \mathrm{Hz}$. 